\title{
Motor für den kontinuierlichen Verbesserungsprozeß
}

\begin{abstract}
Wenn nach der erfolgreichen Einführung eines Umweltmanagementsystems die offensichtlichen ökologisch - ökonomischen Verbesserungspotentiale realisiert worden sind, müssen neve Umweltziele angestrebt werden. Weitere, anspruchsvollere Potentiale können über die systematische Analyse und Steverung der innerbetrieblichen Stoff - und Energieströme erschlossen werden. Hier eignen sich insbesondere Umweltkennzahlen. Möglichkeiten und Grenzen dieses Instruments werden im folgenden anhand von Praxisbeispielen dargestellt.
\end{abstract}

\section{S}

Von Thomas Loew, Jens Clausen und Heinz Kottmann eröffentlicht, um bereits erzielte Fortschritte zu dokumentieren. Die systematische Verwendung als internes Steuerungsinstrument ist bis heute jedoch noch die Ausnahme. In zahlreichen Veröffentlichungen und Diskussionen wurde der Schwerpunkt auf die Gliederung des Umweltkennzahlensystems gelegt und eine Vielzahl von Kennzahlen vorgeschlagen, während die Vorgehensweise bei der Einführung und der praktischen Anwendung im Hintergrund stand (1). Im Auftrag der Baden-Württembergischen Landesanstalt für Umweltschutz erstellte und implementierte das IÖW in drei Pilotunternehmen Umweltkennzahlensysteme(2). Diese dienen in erster Linie der internen Planung und Steuerung, während die Nutzung der Kennzahlen für externe Kommunikation nur eine untergeordnete Rolle spielt.

\section{Vorgehen beim Aufbau eines Umwelfkennzahlensystems}

Das zentrale Ergebnis des Projekts ist eine allgemein übertragbare Vorgehensweise für den effizienten Aufbau eines betrieblichen Umweltkennzahlensystems (Vgl Abb.1).

Ausgangspunkt ist die Bildung eines Projektteams mit engagierten Mitarbeitern aus verschiedenen Abteilungen. Seine erste Aufgabe besteht darin, in einem moderierten Prozeß die wichtigen Umweltfragen des Unternehmens zu ermitteln und sie hinsichtlich ihrer Bedeutung zu gewichten. Dabei werden in der Regel keine neuen Umweltaspekte angesprochen, die untersuchen. Gleichzeitig wird bei der versuchsweisen Erhebung festgestellt, welche Kennzahlen sich tatsächlich bilden lassen und daher für ein Kennzahlensystem geeignet sind. Schließlich sind die für die Ermittlung der Daten und für die Erstellung und Verbreitung erforderlichen Abläufe zu organisieren und gemeinsam mit den Kennzahlen zu dokumentieren. Diese Vorgehensweise hat sich in den Pilotunternehmen bewährt und bildet eine praxisnahe Ergänzung des BMU/ UBALeitfadens zu Umweltkennzahlen (3).

Als gute Managementpraxis im Umgang mit Umweltkennzahlen ist festzuhalten, daß Umweltkennzahlen regelmäßig erhoben, Ziele gesetzt, eine Abweichungskontrolle durch einen Soll-Ist-Vergleich erfolgt und entsprechende Korrekturmaßnahmen durchgeführt werden. Um Unternehmen bei der selbständigen Einführung eines Umweltkennzahlensystems zu unterstützen wird zur Zeit vom IÖW eine Arbeitshilfe erstellt (4). Die folgenden Beispiele zeigen den praktischen Nutzen von Umweltkennzahlen.

\section{Sommerspitzen bei Hansgrohe}

Bei dem Armaturenhersteller Hansgrohe (Offenburg) wurden im Rahmen der Erprobung des ersten Kennzahlensatzes die Monatswerte für den absoluten und den relativen Stromverbrauch in der Kunststoffspritzerei ermittelt. Als Bezugsgröße für den relativen Stromverbrauch wurde die verarbeitete Menge verspritzten Granulats zugrunde gelegt. Während die absoluten Werte über das Jahr gleichmäßig ohne auffällige Schwankungen verlaufen, zeigen die relativen Werte eine auffällige Spitze im Sommer. Die Erklärung lag schnell auf der Hand: In den erheben, um ihre tatsächliche Eignung näher zu

Vorgehensweise

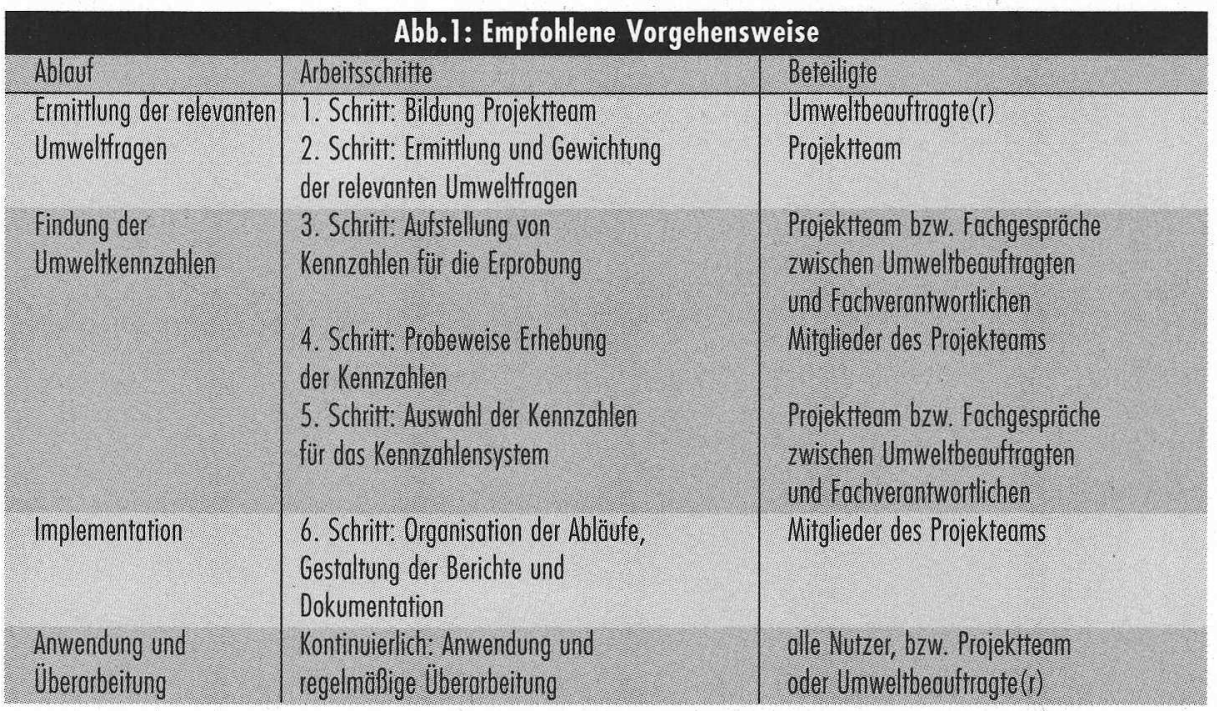


Urlaubsmonaten werden geringere Stïckzahlen gefertigt als im Normalbetrieb, was offensichtlich aber nicht mit einem entsprechend geringeren Stromverbrauch einhergeht.

Man geht davon aus, daß die Maschinen in dieser Zeit häufig leer laufen. Inzwischen wird das Problem näher untersucht. Der zuständige Umweltbeauftragte hofft, daß man diese auslastungsbedingte Überschreitung des durchschnittlichen relativen Stromverbrauchs um etwa die Hälfte reduzieren kann. Damit würde eine Einsparung von jährlich 500 MWh realisiert. Seit Mitte 1998 werden Energiekennzahlen für alle Produktionsabteilungen regelmäßig ermittelt. Es wird davon ausgegangen, daß hier weitere Einsparpotentiale aufgedeckt werden. Die neuen Umweltkennzahlen werden dabei in den bereits bestehenden kontinuierlichen Verbesserungsprozeß (KVP) eingebunden.

\section{- Logistik-Kennzahlen bei Bardusch}

Der Textilmietdienstleister Bardusch (Ettlingen) fährt seine Kunden in festen regelmäßigen Touren an. Vor dem Hintergrund des Strategiewechsels von einer regionalen Versorgung hin $\mathrm{zu}$ einer flächendeckenden Versorgung wurde vermutet, daß sich die Effizienz der Auslieferung verschlechtern könnte. Auf der Basis der vorhandenen umsatzbasierten Kennzahl konnte diese Vermutung nicht bestätigt werden. Zwar nahm die Laufleistung aufgrund der flächendeckenden Versorgung $\mathrm{zu}$, doch wurde gleichzeitig der Umsatz durch Rahmenverträge von Fillialunternehmen gesteigert. Es konnte immerhin eine Steigerung des Umsatzes pro bearbeiteter Menge von 10,5 Prozent erzielt werden.

Mittels der neu aufgestellten Umweltkennzahlen wurden nun aber die realen Auslastungs- und Effizienzveränderungen offenkundig. Die bisher nicht betrachteten Kennzahlen Strecke pro bearbeiteter Menge mit einer Zunahme von 17,5 Prozent und (Container-)Stellplatzauslastung mit einer Abnahme von 10 Prozent verdeutlichten die Auswirkungen auf die realen Vorgänge im Logistikbereich. Die Auslastung der Fahrten hat sich offensichtlich deutlich verschlechtert, wodurch sowohl die Umweltbelastungen als auch die Kosten angestiegen sind.

In einer Bereichsleitersitzung wurden diese Aspekte diskutiert und Lösungsmöglichkeiten gesucht. Ein Ansätze ist, die Verkaufsstrategie darauf auszulegen, daß entlang der Strecken der weiten Touren neue Kunden gewonnen werden, und bei der Fahrzeugauswahl darauf zu achten, daß die Größe und Art der Fahrzeuge auf die Touren abgestimmt werden.

\section{Mitarbeiterbeteiligung bei Georg Kohl}

Wie die Umweltbeauftragte der Firma Georg Kohl (Brackenheim) betont, lebt das Arbeitssicherheits-, Qualitäts- und Umweltmanagement von der Mitarbeit und Motivation der Mitarbeiter. So hat man hier schnell erkannt, daß es nicht ausreicht, die Umweltkennzahlen ohne weitere Erklärungen und Interpretationen zu veröffentlichen, wenn sie als Instrument für den kontinuierlichen Verbesserungsprozeß benutzt werden sollen. Unter dem Motto „Sparen und bewahren“ wurde daher im Juni 1998 eine ,Aktionswoche Energie" mit allen Mitarbeitern durchgefuihrt (Logo).

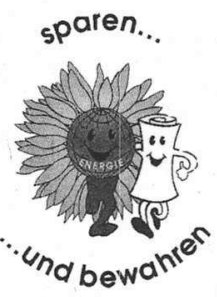

Die Umweltkennzahlen wurden bis dahin so aufbereitet, daß sie jedem Mitarbeiter zugänglich und verständlich sind. Es wurden Vergleiche angestellt, um das Verständnis für Größenordnungen und Relevanz zu fördern, z.B. „Eine Kilo-wattstunde entspricht 350 Gramm Steinkohle und einem Kilogramm Kohlendioxid." Gemeinsam mit einer Fragebogenaktion zum Energieverbrauch wurde eine Energieverbrauchsanalyse erstellt und neue Einsparungspotentiale aufgedeckt.

\section{Grenzen von Umweltkennzahlen}

Neben der in Literatur und Praxis viel diskutierten Flut an Kennzahlen gibt es in den Unternehmen eine beträchtliche Menge an Kennzahlen, die aus organisatorischen oder technischen Gründen nicht oder nur mit unverhältnismäßigem Aufwand gebildet werden können. Beispielsweise konnte die Auslastung der Lkw-Touren nicht direkt per EDV bestimmt werden. Die damit erforderlichen Abschätzungen oder manuellen Erfassungen hätten sowohl die Güte der Kennzahl in Frage gestellt als auch einen zu hohen Aufwand bedeutet. In solchen Fällen stößt das Instrument Umweltkennzahl an praktische Grenzen. Die in zwei Unternehmen geplante Erweiterung der automatischen Betriebsdatenerfassung dürte in Zukunft die Datenlage für die Bildung weiterer Kennzahlen deutlich verbessern.
Die immer wieder diskutierte Vorstellung von einem branchenübergreifenden Umweltkennzahlensystem muß aufgegeben werden. Unterschiedliche Stoff- und Energieströme, Fertigungsstrukturen und Managementstrukturen der Unternehmen machen die Erstellung von betriebsindividuellen Kennzahlensystemen zwingend erforderlich. Selbst bei Unternehmen einer Branche werden je nach Verfahren, Produkte und eingesetzter Stoffe die Umweltkennzahlensysteme unterschiedlich zu gestalten sein. Dies schließt jedoch unternehmensübergreifende Vergleiche nicht grundsätzlich aus. Vielmehr wird deutlich, daß für ein Benchmarking mit Hilfe von Umweltkennzahlen neue, zusätzliche Aufgaben gelöst werden müssen.

\section{Anmerkungen}

(1) Vgl. z.B. Loew, Thomas, Hafdis Hjalmarsdottir: Umweltkennzahlen für das betriebliche Umweltmanagement. IÖW-Schriftenreihe 99/96, Berlin 1996,

Loew, Thomas, Heinz Kottmann, Jens Clausen: Entwicklungsstand von Umweltkennzahlen und Umweltkennzahlensystemen in Theorie und Praxis. IÖW-Diskussionspapier 40/97, Berlin 1997,

Clausen, Jens: Umweltkennzahlen als Steuerungsinstrument für das nachhaltige Wirtschaften von Unternehmen. In: Seidel, Eberhard; Jens Clausen; Eberhard Seifert: Umweltkennzahlen. München, Vahlen 1998, S. 33-70.

ISO/ DIS 14031: Environmental Management - Environmental Performance Evaluation - Guidelines, NAGUS-AA 5 Nr. 03-98 vom 8.1.1998.

(2) Vgl. Kottmann, Heinz; Jens Clausen; Thomas Loew: Zielorientiertes Umweltmanagement mit Hilfe von Umweltkennzahlen, Wissenschaftlicher Endbericht des gleichnamigen Forschungsprojekts. Veröffentlichung voraussichtlich Ende 1998; in: Diskussionsforen des Projekts angewandte Ökologie (PAÖ) 1998, http://www.bwplus.de/paoe/disk98/diskpaoe98.htm

(3) Bundesumweltministerium, Umweltbundesamt ( $\mathrm{Hg}$.): Leiffaden betriebliche Umweltkennzahlen. Bonn, Berlin 1997 (4) Neben der proxisgerechten Beschreibung der Vorgehensweise werden einige Instrumente (Charts, Kennzahlenberichte, Tabellen) als vorbereitete Dateien im Internet zur Verfügung gestellt. Zu finden ab Anfang 1999 unter www.uis-extern.um.bwl.de/ffu/abt2/ocudit.

\section{Die Autoren}

Thomas Loew, Jens Clausen und Heinz Kottmann sind wissenschaftliche Mitarbeiter im Forschungsteld Ökologische Unternehmenspolitik des IÖW Kontakt: IÖW, Giesebrechtstr. 13, 10629 Berlin. T el. $030 / 884594-0$, Fax 030/882 5439 , E-mail: Thomas.Loew@ioew.b.eunet.de 
(c) 20I0 Authors; licensee IÖW and oekom verlag. This is an article distributed under the terms of the Creative Commons Attribution Non-Commercial No Derivates License (http://creativecommons.org/licenses/by-nc-nd/3.o/), which permits unrestricted use, distribution, and reproduction in any medium, provided the original work is properly cited. 